\title{
Are there differences among Bacillus Calmette-Guérin (BCG) strains regarding their clinical efficacy in the treatment of non-muscle invasive bladder cancer? The jury is still out but the answer is likely no
}

\author{
Guan Hee Tan ${ }^{1}$; Cynthia Kuk ${ }^{1,2}$; Alexandre R. Zlotta ${ }^{1,2}$ \\ ${ }^{1}$ Division of Urology, Department of Surgical Oncology, University Health Network, Toronto, ON, Canada; \\ ${ }^{2}$ Division of Urology, Department of Surgery, Sinai Health System, Toronto, ON, Canada
}

Cite as: Can Urol Assoc J 2019 July 23; Epub ahead of print. http://dx.doi.org/10.5489/cuaj.5923

Published online July 23, 2019

$* * *$

\section{Introduction}

The use of Bacillus Calmette-Guerin (BCG) as an intravesical instillation has been a mainstay in the prevention of recurrences as well as progression for non-muscle invasive bladder cancer (NMIBC) and in particular, for high risk disease ${ }^{1,2}$. Whether all BCG strains are equal, or some are more equal than others, as questioned by Noon et $\mathrm{al}^{3}$, has become critically relevant at a time of shortages in BCG supply. In Canada, BCG has been supplied by a limited number of companies (currently Merck only). With recent and previous shortages, whether the use of other strains of BCG for prophylaxis would result in similar outcomes is critical in order to increase the availability of BCG for patients and clinicians. We have reviewed the literature both in the tuberculosis as well as in NMIBC fields. We conclude that, despite differences in genetic composition and immune responses induced, there is no robust evidence that different BCG strains would be associated with different outcomes. This holds true in terms of either protection against tuberculosis or for prevention of recurrence or progression of NMIBC.

It's worth remembering that when mycobacterium bovis underwent the process of attenuation from 1908 to 1921 to become BCG, it lost one of the key regions, RD1. This was a critical step in it's attenuation of the mycobacterium ${ }^{4,5}$. This loss of RD1 is common to all BCG vaccines strains, but these then went on to undergo a series of genomic alterations over time ${ }^{6}$. The various BCG strains can be grouped into early or late strains as shown on figure 1 when they branched off the original BCG. Whether the genomic differences between the early strains (Japan, Moreau and Russia), and the late strains (Frappier, Connaught, Tice, Pasteur, and Phipps) induce different immune responses and protection against tuberculosis is obviously a very relevant question. We as urologists should not forget that BCG was initially introduced to fight a 
life threatening disease affecting millions of men and women in the early $20^{\text {th }}$ century, namely tuberculosis.

BCG vaccination against tuberculosis (the 6 approved strains by the WHO are Pasteur 1173 P2, the Danish 1331, the Glaxo 1077 -derived from the Danish strain, the Tokyo 172-1, the Russian BCG-I, and the Moreau RDJ strains) induces fairly different immune responses according to each particular strains ${ }^{7}$. This may or may not be in keeping with the broad variability in the protection against tuberculosis, which can vary from as low as $0 \%$ to $80 \%{ }^{8,9}$. Recent large reviews of randomized control trials didn't support this hypothesis. The key message gathered from the tuberculosis literature is that despite obvious genetic variations and immune response in the host, especially between early and late strains, no study has shown the superiority of one strain over the other ${ }^{7}$.

In bladder cancer, the influence of BCG regimen, schedules, doses and less often strain on outcomes has been investigated. Direct comparisons between BCG strains are generally lacking. Only one single randomized study ${ }^{10}$ compared head to head in a small cohort two different strains. Interestingly enough, although there was a difference in terms of recurrence free survival of a particular strain over another, the progression rate was actually not statistically different between the Connaught and the Tice strains ${ }^{10}$. Obvious differences between in vitro observations and clinical outcomes exist. For instance, in vitro the Connaught and Russian strains showed the highest direct anti-tumor effects among different BCG strains ${ }^{11}$.

Reviews focusing on this aspect have concluded that despite established genetic differences between strains used in the prevention of NMIBC recurrence and progression, no study supported the superiority of one strain over the others ${ }^{12,13,14}$. Interestingly enough, whole genome sequencing studies taught us that early BCG strains like the Russian strain (used in India in NMIBC), displayed a complete stability of the vaccine substrate genome, whether these were investigated on lots from 1963-1982 or more recently in 2006 ${ }^{15}$.

As a summary, not only several of these BCG strains have maintained their stability at the genome level, but differences which are well known between strains never translated either as an anti-tuberculosis vaccine or in NMIBC in meaningful clinical differences. We believe that part of the explanation comes from the mechanism of action of BCG in the prophylaxis of bladder cancer recurrences/progression ${ }^{16,17}$. One should never forget that the doses we instill in the bladder are astronomical compared to the anti-tuberculosis vaccine. BCG are live attenuated mycobacteria made of numerous subcomponents which are both antigenic and able to induce very strong cellular immune responses ${ }^{16}$. They key word as often in biology is redundancy. Many subcomponents of the BCG, irrespective of the strain, are able to drive a robust and necessary TH1 response. This ultimately will lead to the elimination of tumor cells. Because so many components, both from the BCG wall, membrane or secreted by the live attenuated mycobacteria ${ }^{16}$ are able to unleash the immune cascade ultimately resulting in tumor cell killing, 
it is likely, given all the evidence which is available, from the genetics, epidemiology, and the clinical perspective that the variability in sub-strains does not play any major role, if any at all.

We live at a time of shortages in BCG which may affect patients' management and when patients are in dire need of these intravesical treatments. The available evidence does not suggest that the introduction in the Canadian or North American market of established BCG strains in other countries, which have produced adequate prophylaxis in NMIBC, would be in any way harmful or detrimental to the wellbeing of Canadian patients. Ideally though, more research to show equivalence between strains should be encouraged as only one randomized study with a limited number of patients has addressed this specific point. 


\section{References}

1. Babjuk M, Burger M, Comperat E, et al. EAU guidelines on non-muscle-invasive bladder cancer (TaT1 and CIS).; c2018. https://uroweb.org/wp-content/uploads/EAUGuidelines-Non-muscle-invasive-Bladder-Cancer-TaT1-CIS-2018.pdf. Accessed January 17, 2019.

2. Kassouf W, Traboulsi SL, Kulkarni G, et al. CUA guideline on the management of nonmuscle invasive bladder cancer. Can Urol Assoc J 2015;9:E690-704.

3. Noon AP, Kulkarni GS. All bacillus Calmette-Guérin (BCG) strains are equal, but some BCG strains are more equal than others. Eur Urol 2014;66:689-91.

4. Abdallah AM, Hill-Cawthorne GA, Otto TD, et al. Genomic expression catalogue of a global collection of BCG vaccine strains show evidence for highly diverged metabolic and cell-wall adaptations. Sci Rep 2015;5:15443.

5. Abdallah AM, Behr MA. Evolution and Strain Variation in BCG. Adv Exp Med Biol 2017;1019:155-69.

6. Behr M. BCG--different strains, different vaccines? Lancet Infect Dis. 2002 Feb;2(2):86-92.

7. Ritz N, Hanekom WA, Robins-Browne R, et al. Influence of BCG vaccine strain on the immune response and protection against tuberculosis. FEMS Microbiol Rev 2008;32:821-41.

8. Trunz BB, Fine P, Dye C. Effect of BCG vaccination on childhood tuberculous meningitis and military tuberculosis worldwide: a meta-analysis and assessment of costeffectiveness. Lancet 2006;367:1173-80.

9. Roy A, Eisenhut M, Harris RJ, et al. Effect of BCG vaccination against Mycobacterium tuberculosis infection in children: systematic review and meta-analysis. BMJ 2014;349:g4643.

10. Rentsch CA, Birkhäuser FD, Biot C, et al. Bacillus Calmette-Guérin strain differences have an impact on clinical outcome in bladder cancer immunotherapy. Eur Urol 2014;66:677-88.

11. Secanella-Fandos S, Luquin M, Julián E. Connaught and Russian strains showed the highest direct antitumor effects of different Bacillus Calmette-Guérin substrains. J Urol. 2013;189(2):711-8.

12. Chou R, Selph S, Buckley DI, et al. Intravesical Therapy for the Treatment of Non muscle Invasive Bladder Cancer: A Systematic Review and Meta-Analysis. J Urol 2017;197:1189-99.

13. Gan C, Mostafid H, Khan MS, et al. BCG immunotherapy for bladder cancer--the effects of substrain differences. Nat Rev Urol 2013;10:580-8.

14. Boehm BE, Cornell JE, Wang H, et al. Efficacy of bacillus Calmette-Guérin Strains for Treatment of Non muscle Invasive Bladder Cancer: A Systematic Review and Network Meta-Analysis. J Urol 2017;198:503-10.

15. Sotnikova EA, Shitikov EA, Malakhova MV et al. Complete Genome Sequence of Mycobacterium bovis Strain BCG-1 (Russia). Genome Announc 2016;4(2). 
16. Zlotta AR, Van Vooren JP, Denis O, et al. What are the immunologically active components of bacille Calmette-Guérin in therapy of superficial bladder cancer? Int $\mathrm{J}$ Cancer 2000;87(6):844-52.

17. Redelman-Sidi G, Glickman MS, Bochner BH. The mechanism of action of BCG therapy for bladder cancer--a current perspective. Nat Rev Urol 2014 Mar;11(3):153-62.

18. Bottai D, Brosch R. The BCG Strain Pool: Diversity Matters. Mol Ther 2016;24(2):201203 


\section{Figures and Tables}

Fig. 1. Early and late strains of BCG. ${ }^{18}{ }^{*}$ Approved BCGs by the World Health Organization (WHO).

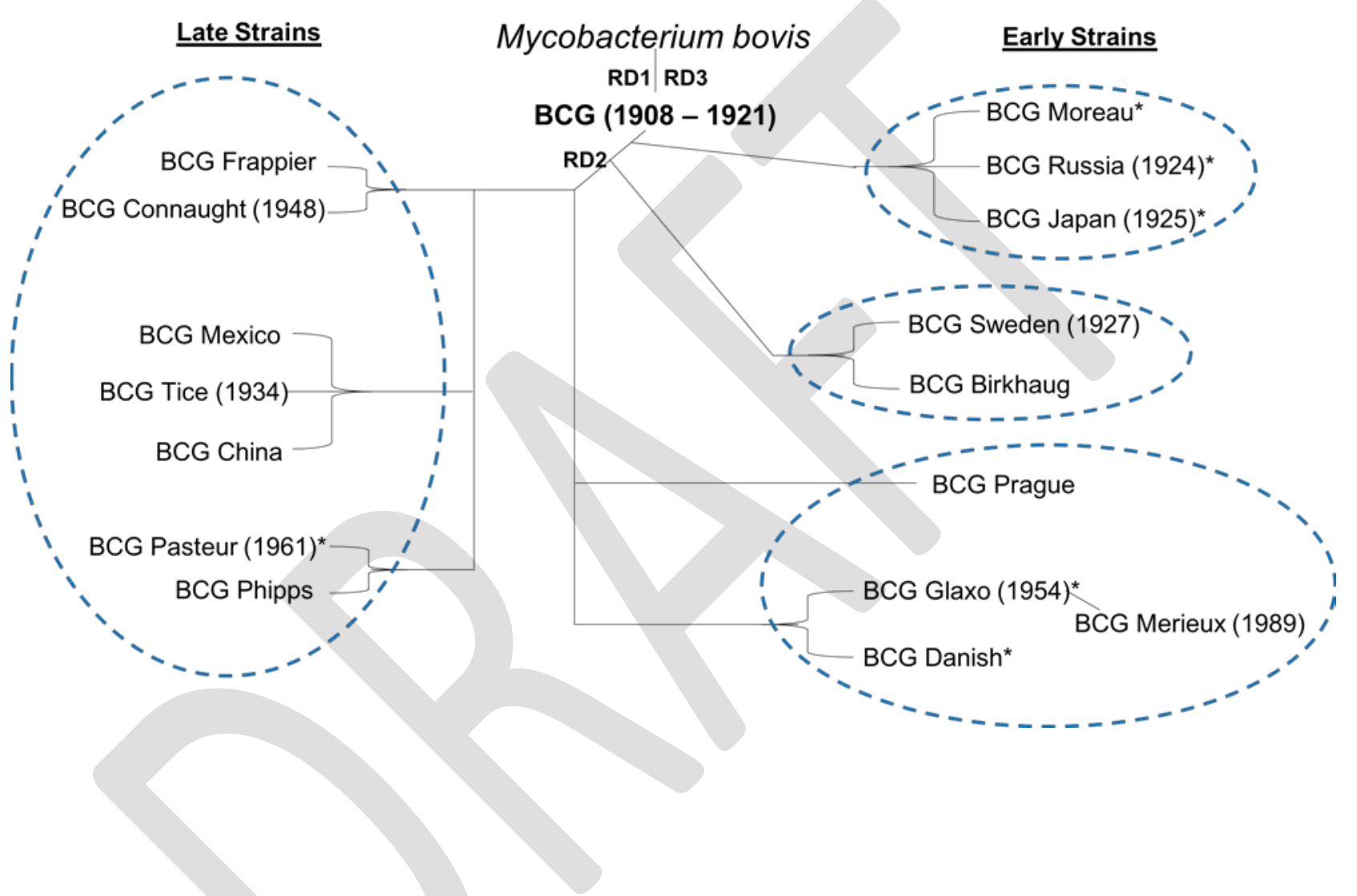

